\title{
Overcoming the Challenges of Epilepsy in Nepal- From Grassroots to Advanced level
}

\author{
Sharad Hari Gajuryal ${ }^{1}$, Shreejana Maharjan ${ }^{2}$, Koji lida $^{3}$, Basant Pant ${ }^{4}$ \\ ${ }^{1}$ Department of Public Health, Annapurna Neurological Institute \& Allied Sciences, Maitighar, Kathmandu, Nepal \\ ${ }^{2}$ Department of Psychiatry, Annapurna Neurological Institute \& Allied Sciences, Maitighar, Kathmandu, Nepal \\ ${ }^{3}$ Department of Neurosurgery, Annapurna Neurological Institute \& Allied Sciences, Maitighar,Kathmandu, Nepal \\ ${ }^{4}$ Epilepsy Center, Department of Neurosurgery, Hiroshima University Hospital ,Hiroshima, Japan
}

\section{CORRESPONDENCE}

Dr. Sharad Hari Gajuryal

Public Health Director

Annapurna Neurological Institute \& Allied

Sciences, Maitighar, Kathmandu

Email : sharad2005@hotmail.com

\section{ARTICLE INFO}

Article History

Submitted: 01 November, 2020

Accepted: 15 December, 2020

Published: 8 February,2021

Source of support: None

Conflict of Interest: None

\begin{abstract}
Epilepsy is considered to be one of the common neurological disease with a high public health concern in recent years. The burden of epilepsy is also high affecting the patients and their care givers physically, mentally as well as socio economically. This burden seems very high in low- and middle -income countries with high rate of treatment gap resulting in higher number of morbidity and mortality. There are various challenges in low-middle income countries to overcome the treatment gap. Sustained and coordinated action prioritizing epilepsy from the rural setting to the advance setting can help in tackling the challenges. This article focuses on the challenges of epilepsy treatment in low-middle income country like Nepal and discusses the ways forward to overcome it.
\end{abstract}

Keywords: Epilepsy; Treatment Challenges; Nepal.

\section{INTRODUCTION}

Epilepsy is a global public health problem affecting people of all ages, sex, races, nations and social class. Nearly $80 \%$ of people with epilepsy live in low and middle-income countries within limited resources where the rate of new cases is up to two fold higher than that of high-income countries.${ }^{1}$ The estimated proportion of the worldwide general population with active epilepsy at a given time is between 4 and 10 per 1000 people; however, in low and middle-income countries, the proportion is much higher, ranging between 7 and 14 per 1000 people. ${ }^{2}$ Epilepsy accounts for $0.75 \%$ of the global burden of disease, that combines years of life lost due to premature mortality and time lived in less than full health. ${ }^{3}$ It is particularly challenging for countries that are often termed resource poor because they constitute a large portion of the general (85\%) and epilepsy (75.0\%) populations and their own health expenditure constitutes only a minor share $(12.0 \%)$ of global health spending . ${ }^{4}$

In a low income county like Nepal, where still out of the pocket expenditure exists, economic factor is also one of the major cause of treatment gap. Beside these, other challenges in epilepsy treatment includes prioritization of resources and funding, access to health care facilities, underlying etiology of epilepsy, expertise and diagnostic capacity of health care personnel, treatment options, sociocultural attitudes toward epilepsy, stigmatization, misconceptions, discrimination and treatment-seeking behavior. ${ }^{1,5}$ In many countries, various projects have been carried out and strategies have been adopted to reduce the treatment gap and morbidity of people with epilepsy, to train and educate health professionals, to dispel stigma, to identify potential prevention strategies, and to develop models integrating epilepsy care into local health systems ${ }^{3,5,6}$ In resource limited country like Nepal, various implications from these kind of projects can be applied so as to overcome the existing challenges faced by people with epilepsy in terms of comorbidity, mortality, and stigmatization, as well as the barriers to treatment engendered by resource prioritization and cultural beliefs. This articles aims to provide the mapping of sustained and coordinated programs from grass root level to policy level which is to be prioritized by individual institution as well as government.

\section{Dimension of Challenges}

Treatment Gap : Treatment gap in epilepsy is defined as the percentage of people with active epilepsy who are not being appropriately treated (either as a result of lack of access to treatment or of being on inadequate treatment) 
in a given population at a given time ${ }^{7}$ The treatment gap is the most common problem for epilepsy management in the low-income regions of Asia with the percentage of treatment gap more than $70 \%$ in Nepal. ${ }^{1}$ Affordability, accessibility and availability of care and treatment is the major determinant of the treatment gap the treatment gap. Various reasons for treatment gap in epilepsy compromises accessibility of adequate health care provision, availability of specialist doctor, unavailability of Anti-epileptic drugs, lack of affordable treatment, distance to health care facilities, lack of awareness of epilepsy, stigmatization, cultural beliefs, and trust in traditional treatment. $^{7}$

Access to Epilepsy Specialist: Because of the lack of Neurologist \& Neurophysicians, Epilepsy in Nepal is frequently managed by primary care physicians, There are only around 80 Neurosurgeons and 20 neurologist in Nepal compromising of 0.3 neurology specialist in 100000 populations. The median number of neurologists per 100 000 population is 0.03 for low-income countries compared with 2.96 for high-income countries. ${ }^{8}$ High class imaging technology is available only in city areas and the epilepsy is diagnosed mostly on the basis of clinical information only. Therefore, morbidity and mortality associated with epilepsy may be increased because of failure to correctly diagnose people with epilepsy in Nepal.

Access to Anti-Epileptic Drugs: In developing countries, availability and quality of drugs are critical factors for effective management and control of epilepsy. Commonly used conventional antiepileptic drugs for pharmacotherapy in epilepsy in Nepal are phenytoin, carbamazepine and valproic acid. The availability of the selected essential medicines is found to be ununiform and insufficient in various part of Nepal. In the private pharmacies, High competition in the products with high price variation, and poor access to cost effective brand is often seen. ${ }^{9,10}$ Majority of the patients should take multiple drugs with out-of-pocket expenditure in Nepal making economic burden to the patient as well as care giver. These is further lack of regulations for further availability of antiepileptic medicines.

Lack of National Registry in Epilepsy: Nepal lacks adequate data on epilepsy. There is still no any clear information amout the exact prevalence of the epilepsy in Nepal. Fewer number of prevalence study in epilepsy in community and individual hospitals in the past may not generalize the exact prevalence of epilepsy. There is no specific National registry for recording the EEG from various health care Institutions that can definitely enable operational development and improvements in the quality, cost-effectiveness and geographic equality of care.
Government Prioritization: Nepal is a part of various epilepsy Association including International League for Epilespy Association ( ILAE) having its own National Association, Nepal Epilepsy Association ( NEA), only fewer works have been done to prevent and priortise eplilepsy. Nepal is witness of various epilepsy declaration but the progress is very nominal on it. Nepal government have various plans and policies for different non-communicable disease but there is no any structured policy to combat epilepsy in Nepal. This may be a cause for treatment gap and prevention of epilepsy in Nepal. Other policies like epilepsy treatment protocol as well as restriction of motor driving by patient with epilepsy, involvement of social workers for patient with eplipsy and vocational training for epilepsy patient still lacks in Nepal.

Recognition of Epilepsy Center: Few of the higher institution in Nepal do have best specialist as well as treatment protocol including epilepsy surgery in Nepal. These type of centers must be acknowledged by Ministry of Health as well as Medical Association in Nepal as an Epilepsy center. Majority of the patient from rural area do not have idea about where to seek for proper care and treatment for epilepsy. Lack of recognition of the institute fulfilling the international criteria as epilepsy center by concerned authority may divert the patient to choose to right center for epilepsy treatment.

Research: Limited researches have been conducted in Nepal in epilepsy including the community survey, KAP survey in epilepsy and patients profile with epilepsy. The results are only limited to fewer area and couldn't be generalized to the whole population as these result only may indicate the tip $f$ the iceberg. Nepal lacks the research center for conducting molecular and genetic research for epilepsy. A nation wide research on various non communicable disease have been conducted by Nepal Health Research council but it lacks the data on epilepsy.

Multi-sectoral collaboration: Prevention, management and rehabilitation of epilepsy is not only the responsibility of a single authority and organization but it's a multisectoral collaboration to combat epilepsy specially in low-middle income countries. A joint effort of health professionals NGO, INGO and various collaborating organization for health is a crucial need for the country like Nepal to combat Epilepsy. Nepal still lacks the plan for priortising epilepsy as a public health agenda.

\section{Ways Forward}

Epilepsy in Nepal still remains a big challenge. The high treatment gap, the widespread use of traditional healers for epilepsy, and the poor understanding of epilepsy 
among the epilepsy patients and social stigma demands greater efforts in public and patient education. Various study done in the country with higher treatment gap have already shown that training to health professionals as well as awareness to the patients have decreased treatment gap in public. ${ }^{5,11,12} \mathrm{~A}$ multisectoral collaboration to educate the local health care professionals about epilepsy and awareness to public about epilepsy is the need of the hour. The major cause of Epilepsy in Nepal is Neurocysticersosis which can be reduced by preventive awareness and education. Furthermore, establishment of a national registry on epilepsy which would help in prevalence survey as well as clinical and basic research in the epilepsy affected area should be prioritized.

Epilepsy should also be encorporated in government policy as a public health disease with social and economical support to the patient with epilepsy. Policy on Cost effective generic drug distribution and its availability is also one of the effective way for easy accessibility to epilepsy medicine is also one the best way to reduce the treatment gap. Recognition of the Institute as epilepsy center that meets international criteria will also probably leads the patients and primary health care workers for further referral. These type of sustained and coordinated action to prioritize epilepsy in public health agendas addressing accessibility, and gaps in knowledge, care, treatment and research is required in the low-middle income countries and resource constrained setup which may finally reduce the prevalence of epilepsy as well as treatment gap .

\section{REFERENCES}

1. Trinka E, Kwan P, Lee B, Dash A. Epilepsy in Asia: Disease burden, management barriers, and challenges. Epilepsia [Internet]. 2019 Mar 1 [cited 2020 Feb 16];60(S1):7-21. Available from: http://doi.wiley.com/10.1111/epi.14458

2. Radhakrishnan A. Bridging the treatment gap in epilepsy-is there an emerging trend in the use of newer antiepileptic drugs? Neurol India [Internet]. 2016 Nov 1 [cited 2020 Feb 16];64(6):1140. Available from: http://www. neurologyindia.com/text.asp?2016/64/6/1140/193773

3. Epilepsy [Internet]. World Health Organisation. 2016 [cited 2020 Feb 16]. Available from: https://www.who.int/newsroom/fact-sheets/detail/epilepsy

4. Kimberly P, Kenneth S, Robert M. McCarthy's Introduction to Health Care Delivery: A Primer for Pharmacists - Google Books [Internet]. 2016 [cited 2020 Feb 16]. 487 p.

5. Bhalla D, Samleng C, Gérard D. Epilepsy in Asia: A Cambodian Experience. Neuroepidemiology, 40(4), 260263 | 10.1159/000345118. Neuroepidemiology [Internet]. 2013 [cited 2020 Feb 16];40(4):260-3.
6. Modern and traditional healers combine forces to improve epilepsy care in Mozambique // International League Against Epilepsy. Epigraph [Internet]. 2019 [cited 2020 Feb 16];21(4). Available from: https://www.ilae.org/journals/ epigraph/epigraph-vol-21-issue-4-fall-2019/modern-andtraditional-healers-combine-forces-to-improve-epilepsycare-in-mozambique

7. Neligan A, Sander JW. The treatment gap in epilepsy: A global perspective. Epileptology. 2013 Mar 1;1(1):28-30

8. Organization WH. NEUROLOGY ATLAS presents for the first time, the most World Federation of Neurology London [Internet]. [cited 2020 Feb 18]. Available from: www.who. int/mental_health

9. Shrestha R, Ghale A, Chapagain BR, Gyawali M, Acharya T. Survey on the availability, price and affordability of selected essential medicines for non-communicable diseases in community pharmacies of Kathmandu valley. SAGE Open Med. 2017 Dec;5:205031211773869.

10. Devkota A, Paudel A, Koirala B, Baral D, Gautam S, Sharma SK. Variation in Price of Medicines and Free Medicine Availability for Treatment of Non-communicable Diseases in Public Sector of Eastern Nepal. J Nepal Health Res Counc. 2018 Jul 5;16(2):118-23.

11. Wang W, Wu J, Dai X, Ma G, Yang B, Wang T, et al. Global campaign against epilepsy: Assessment of a demonstration project in rural China. Bull World Health Organ. 2008 Dec;86(12):964-9.

14. Global Campaign Against Epilepsy out of the shadows International Bureau for Epilepsy World Health Organization International League Against Epilepsy. 